\title{
Hydrophobic Lurylamine Modified Heteropoly Acid as an Efficient and Recyclable Catalyst for the Hydrolysis Reaction in Aqueous Solution under Microwave
}

\author{
Lin $W^{a}$, Bian-Jiang ZHU, and Yin-Feng WU \\ Qingdao Technical College, Qingdao, Shandong Province \\ awul@qtc.edu.cn
}

\begin{abstract}
A new modified phosphotungstic acid (HPW) catalyst (HPW-catal) was synthesized b y a simple mixing method under ambient atmosphere and evaluated for the hydrolysis of sucrose and ethyl acetate under microwave irradiation. The as-synthesized catalyst was characterized by X-ray diffraction (XRD), Fourier transform infrared spectroscopy(FTIR), and NH3-TPD, which proved that HPW was sucessfully modified with lurylamine and the catalyst still remains the Keggin structure. Significantly, the HPW-catal showed excellent catalytic hydrolysis perforance for the sucrose and ethyl acetate under microwave irradiation. Both of the reactions were studied over other solid acid catalysts, such as AM-15 and ZSM-5. At mild tempretures, the sucrose and ethyl acetate could be hydrolyzed into corresponding products. The maximum glucose yield and alcohol could reach $100 \%$ and $95 \%$, respectively. The HPW-catal can be recycled and reused. The HPW-catal will provides new ideas for the modifying of heteropoly acids.
\end{abstract}

\section{Introduction}

Mineral acids $\left(\mathrm{H}_{2} \mathrm{SO}_{4}, \mathrm{HCl}\right.$ and $\left.\mathrm{HNO}_{3}\right)$ are essential in many industrially reactions such as alkylation, esterization and isomerization. Although these acid catalysts always exhibit high activity, they are typically associated with serious problems of high toxicity, corrosion, production of waste, difficulty of separation and recovery. From the viewpoint of "green chemistry" and "green technology", production methods should be refined so as to minimize adverse effects on the environment or human health. Therefore, the search for more environmentally friendly solid acid catalysts had extraordinarily been paid attention. ${ }^{2,3}$ Among these solid acids, Heteropoly acids (HPAs) stand out due to their strong Bronsted acidity and fairly high thermal stability in the solid state. ${ }^{4-6}$ As a consequence, several new industrial processes based on HPAs, such as hydration of olefins, oxidation of methacrolein, and polymeriztion of tetrahyrofuran, have been developed and commercalized. ${ }^{7}$ Additionally, HPAs catalysed Fries rearrangement of phenyl acetate, ${ }^{8}$ and Friedel-Crafts acylation of phenol ${ }^{9}$ and oxidation of alcohols ${ }^{10}$ have also been reported. Therefore, HPAs are excellent heterogenous solid acid catalyst in the catalytic system of solid-gas reaction or hydrophobic solvent.

On the other hand, the catalytic reactions in aqueous systems have interesting advantages including low cost, nontoxicity, availability, and safety. HPAs were also employed as homogeneous catalysts to accomplish a series of reactions in aqueous solution with relative high performance due to their high solubility in the polar solution. For example, in the hydrolysis of cellulose, $\mathrm{H}_{3} \mathrm{PW}_{12} \mathrm{O}_{40}$ (HPW) exhibited high yield and selectivity of glucose $(50.5 \%$ and $>90 \%$ separately) due to its strong Bronsted acid strength and high hydrothermal stability. ${ }^{11}$ The effects of Bronsted and Lewis acidity on the activity and selectivity of the HPA-based catalyst for the hydrolysis of cellulose were also discussed in another reports. $^{12}$ HPAs together with supported ruthenium catalysts showed not only high activity but also remarkable selectivity to sugar alcohols reaching up to $81 \%$ yield of $\mathrm{C} 4$ to $\mathrm{C} 6$ sugar alcohols in aqueous phase with $7 \mathrm{~h}$ at 160 ${ }^{\circ} \mathrm{C} .{ }^{13}$ Although the HPAs are unique acid catalysts in the aqueous reaction, the high solubility in polar reaction media largely hinder their practical utilizations. So far, some efforts that impregnating HPAs onto or encapsulating it into porous carriers with high surface areas, such as silica, active carbon, zeolite, ${ }^{14-17}$ and partially exchanging protons of the parent HPA with large inorganic cations like $\mathrm{Cs}^{+}, \mathrm{K}^{+}, \mathrm{Rb}^{+}$and $\mathrm{NH}_{4}^{+},{ }^{+18}, 19$ have been made to separate the HAPs and reused them. Unfortunately, these methods were seldom successful. So, it is very desirable to develop an efficient and recyclable HPAs catalystic systmem to solve the separation and recycle issues of HPAs catalyst. In our opinion, an ideal catalyst in aqueous solution should mix and contact very well with reactant and separate spontaneously from the system after reaction. 


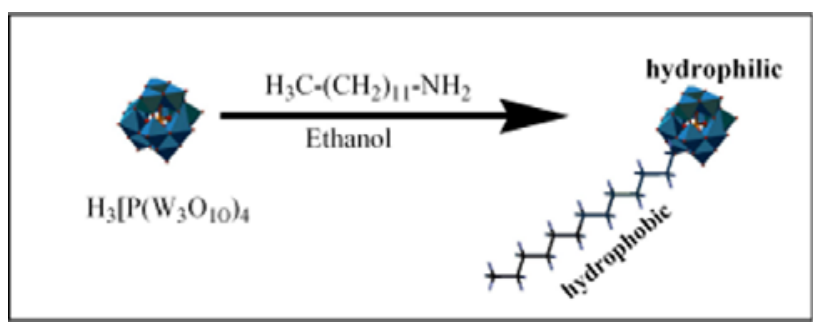

Figure 1. Synthesis of modified HPW catalyst

In this study, a much more environmentally benign and safe process for the hydrolysis of ethyl acetate and sucrose with luryamine modified heteropoly acid is reported. Most importantly, it's much easier than other typical HPAs to separate the catalysts with the products as they exists in different phases. What's more, the modified heteropoly acids showed stable and high catalytic activity. As far as we know, this is the first report of successful heteropoly acid catalyst modified in this way aiming to change its hydrophobicity which still has excellent catalytic acivity.

\section{Experimental}

\subsection{Materials}

Lurylamine(Tianjin Guangfu Fine Chemical Research Institute, lurylamine content $\geq 99 \%$ ), H3PW12O40 (Sinopharm Chemical Reagent Co., Ltd, AR), alcohol (Sinopharm Chemical Reagent Co., Ltd, AR), ethyl acetate (Sinopharm Chemical Reagent Co., Ltd, AR), sucrose (Tianjin Guangcheng Chemical Reagent Co.,Ltd, AR), concentrated sulphuric acid (Sinopharm Chemical Reagent Co., Ltd, AR), dilute sulphuric acids $(0.1 \mathrm{~mol} / \mathrm{L}, 0.3 \mathrm{~mol} / \mathrm{L}$, $0.5 \mathrm{~mol} / \mathrm{L}$, self-made by diluting concentrated sulphuric acid with a certain amount of water), Amberlyst ${ }^{\circledR}-15$ (Abbr.Am-15, SIGMA-ALDRICH), HZSM-5 (Nankai University, silicon / aluminum ratio is 50), ammonium phosphotungstate (Abbr.AWP, Chengdu Kelong Chemical Reagent Factory, AR), distilled water.

All the other assistant chemicals were purchased from Sinopharm Chemical Reagent Co., Ltd, and used as received.

\subsection{Modified HPW Catalysts Preparation}

Under the ambient tempreture, 2 mmol lurylamine was dissolved in $10 \mathrm{~mL}$ alcohol and $1 \mathrm{mmol} \mathrm{HPW}$ was dissolved in $2 \mathrm{~mL}$, then mixed them in a $50 \mathrm{~mL}$ vessel, the mixture was stirred magnetically for $2 \mathrm{~h}$ before adding distilled water to generate white precipitatation. Then the white precipitatation were washed with distilled water for several times using centrifuge and dried overnight at $100{ }^{\circ} \mathrm{C}$ in an oven. The resultant powders were target catalysts called HPW-catal.

\subsection{Catalytic Experiments}

The hydrolysis of ethyl acetate: The reaction was carried out in a CEM Discover microwave synthesis reactor (CEM corporation, USA), after $0.3 \mathrm{~g}$ HPW-catal, $0.3 \mathrm{~mL}$ ethyl acetate were added into $15 \mathrm{~mL}$ distilled water, the solution was heated in $70{ }^{\circ} \mathrm{C}$ and the reaction was stopped after 0.5 $\mathrm{h}, 1 \mathrm{~h}, 1.5 \mathrm{~h}, 2 \mathrm{~h}, 2.5 \mathrm{~h}$, respectively. In comparison to HPW-catal, the hydrolysis of ethyl acetate catalyzed by 0.3 g AM-15, 0.3 g ZSM-5, 0.3 g AWP, $0.1 \mathrm{~mol} / \mathrm{L} \mathrm{H}_{2} \mathrm{SO}_{4}$, $0.3 \mathrm{~mol} / \mathrm{L} \mathrm{H}_{2} \mathrm{SO}_{4}, 0.5 \mathrm{~mol} / \mathrm{L} \mathrm{H}_{2} \mathrm{SO}_{4}$ were also conducted in the same condition as HPW-catal. What's more, the traditional hydrothermal reaction experinments were also performed, in this condition all of the reaction systems were performed in $70{ }^{\circ} \mathrm{C}$ and the time lasted for $1 \mathrm{~h}, 3 \mathrm{~h}, 5$ h, $8 \mathrm{~h}, 10 \mathrm{~h}$, respectively.

The hydrolysis of sucrose: In this experiment, $0.02 \mathrm{~g}$ HPW-catal, $0.02 \mathrm{~g}$ sucrose and $1 \mathrm{~mL} \mathrm{H}_{2} \mathrm{O}$ were mixtured which were heated at different temperatures for different reaction time under the microwave reactor mentioned above. The performance of other catalysts in the same condition were also tested.

The repeatability test: Both of the hydrolysis reactions above were used to test the repeatability of HPW-catal. The former reaction, after $0.3 \mathrm{~g}$ HPW-catal, $0.3 \mathrm{~g}$ ethyl acetate were added into $15 \mathrm{~mL}$ distilled water, the solution was heated in $70{ }^{\circ} \mathrm{C}$ holding for $1.5 \mathrm{~h}$. After the reaction, HPW-catal were separated from the solution, washed with distilled water for several times, and dried in oven. The resulted HPW-catal were used to catalyze again as the same condition above, repeat four times. The later reaction, $0.02 \mathrm{~g} \mathrm{HPW}$-catal, $0.02 \mathrm{~g}$ sucrose and $1 \mathrm{~mL} \mathrm{H}_{2} \mathrm{O}$ were mixtured, then the mixture was heated in $75{ }^{\circ} \mathrm{C}$ holding for $2 \mathrm{~h}$. then the similar operation as the former reaction were carried out.

\subsection{Product Analysis}

Samples in the hydrolysis of ethyl acetate were filtered through $0.22 \mu \mathrm{m}$-pore-size filters (Membrana) prior to analysis. The products in resultant solutions after reaction were identified by gas chromatograph (GC, 7890A, Agilent, USA). The temperature of injection was at $260^{\circ} \mathrm{C}$. Nitrogen was used as the carrier gas, and its flow rate was $1 \mathrm{~mL} / \mathrm{min}$. The injection volume was $1 \mu \mathrm{L}$ and the split ratio was $20: 1$. The temperature program for the column was held at $60{ }^{\circ} \mathrm{C}$ for $1 \mathrm{~min}$ and programmed to $120^{\circ} \mathrm{C}$ at a ramping rate of $25^{\circ} \mathrm{C} / \mathrm{min}$, to $135^{\circ} \mathrm{C}$ at a ramping rate of $30{ }^{\circ} \mathrm{C} / \mathrm{min}$, then to $180{ }^{\circ} \mathrm{C}$ at a ramping rate of $15^{\circ} \mathrm{C} / \mathrm{min}$ with a hold time of $3 \mathrm{~min}$.

Samples in the hydrolysis of sucrose were also filtered through $0.22 \mu \mathrm{m}$-pore-size filters (Membrana) prior to analysis. The yield of glucose in resultant solutions was measured by Glucose biosensor (SBA-40E, Institute of Biology, Shangdong Academy of Science,China). Take 1 $\mathrm{g} / \mathrm{L}$ of glucose solution as standard liquids in order to calibrate the glucose content.

Products yield of the two kinds of reactions were calculated by the following equation:

Products yield $=\mathrm{np} / \mathrm{nr} * 100 \%$

where $\mathrm{np}$ is amount of substance of products quantified in this work and $\mathrm{nr}$ is amount of substance of reactants. 


\subsection{Catalyst Characterization}

FT-IR spectra were recorded on Thermo Electron Fourier transform infrared spectrophotometer (FT-IR) Nicolet 6700 . The sample was pelletized with $\mathrm{KBr}$ and used to record the infrared spectra, ranging from 4000 to $400 \mathrm{~cm}-1$. Powder X-ray diffraction data (XRD) were recorded at a scanning rate of $0.02 \% \mathrm{~s}$ using a Bruker D8 ADVANCE X-ray diffractometer at $40 \mathrm{kV}$ and $40 \mathrm{~mA}$ with monochromatized $\mathrm{Cu} \mathrm{K} \alpha(\lambda=1.5418 \mathrm{~nm})$ radiation. $\mathrm{N}_{2}$ adsorption-desorption experiments were performed with a Micromeritics ASAP $2020 \mathrm{M}+\mathrm{C}$ surface area and porosity analyzer. The samples were outgassed for $10 \mathrm{~h}$ under vacuum at $100^{\circ} \mathrm{C}$ prior to adsorption. $\mathrm{NH}_{3}$-TPD experiments were performed with a Micromeritics Autochem II chemisorption analyzer.

\section{Results and Discussion}

The FT-IR spectra method used to determine the change of groups on the samples before and after synthesis. FTIR spectra of HPW, HPW-catal, lurylamine were shown in Fig. 2. The Keggin structure of both HPW and HPWcatal was identified by four characteristic absorption bands appearing at (i) $1080 \mathrm{~cm}^{-1}$ (stretching frequency of $\mathrm{P}-\mathrm{O}_{\mathrm{a}}$ in the central $\mathrm{PO}_{4}$ tetrahedron), (ii) $983 \mathrm{~cm}^{-1}$ (terminal bands for $\mathrm{WO}_{\mathrm{d}}$ in the exterior), (iii) $895 \mathrm{~cm}^{-1}$ (stretching vibration of $\mathrm{W}-\mathrm{O}_{\mathrm{b}}-\mathrm{W}$ bridges between corner sharing octahedras) and (iv)799 $\mathrm{cm}^{-1}$ (stretching vibrations of $\mathrm{W}-\mathrm{Oc}-\mathrm{W}$ bridges between edge sharing octahedras) as described elsewhere. ${ }^{20,21}$ In spite of the decrease of peak intensities and slight shift of peak positions, the four peaks appeared distinctively, which indicates that the Keggin structure of heteropolyanion was well reserved after the protons in the HPA were substituted by the organic cations. What's more, the another powerful evidence was shown that there were three ranges of Keggin structure feature peaks at $7 \sim 9^{\circ}$, $18 \sim 21^{\circ}, 26 \sim 29^{\circ}$, respectively in XRD pattern, although some peaks became weak and absence due to the reaction between the lurylamine and HPA (in Figure. 3). ${ }^{22}$ The two elliptic dotted lines stand stretching vibration and bending vibration of $\mathrm{C}-\mathrm{H}$ respectively, indicating the sucessful introduction of carbon chain into the HPW-catal. The stretching vibration of $\mathrm{OH}$ for HPW-catal became weak compared with HPW because of the effects of hydrophobic nature of HPW-catal.At the same time, the vabrication of $-\mathrm{NH}_{3}{ }^{+}$between $3100-3335 \mathrm{~cm}^{-1}$ appears after modification, which also indicated that the HPW has been modified by lurylamine. Based on above analysis, the lurylamine molecue has been incorporated onto the structure of HPW. The hydrophobic character of the HPW-catal also can prove this.while the NH stretching vibration blueshifts because of the effect of modification.

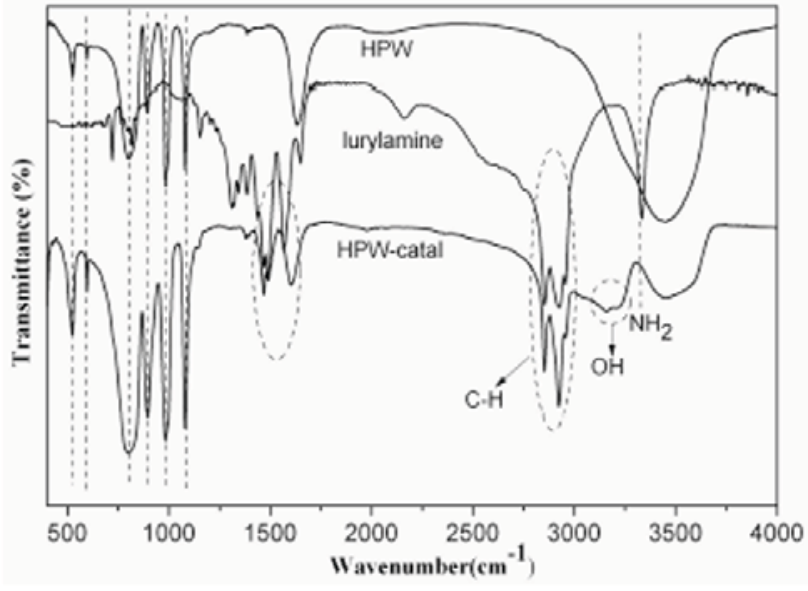

Figure 2. FT-IR spectra of HPW, lurylamine, HPW-catal

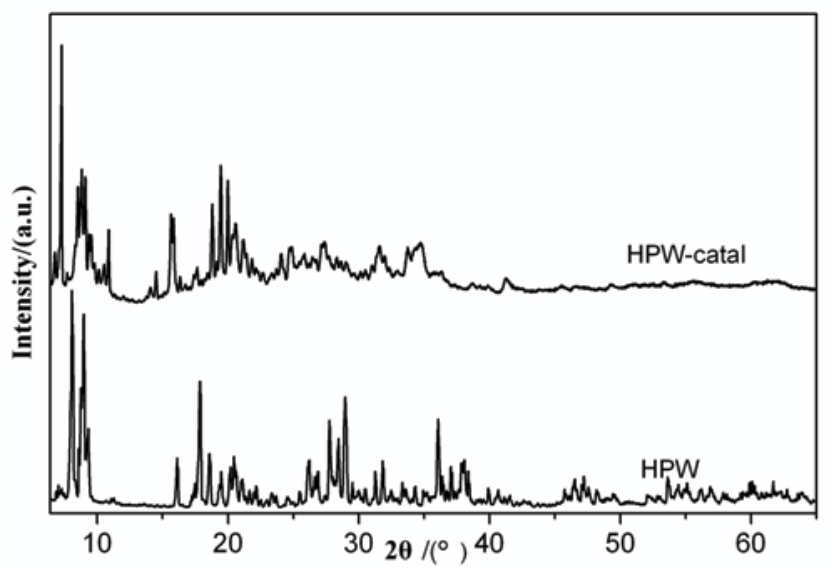

Figure 3. Powder X-ray diffraction patterns of HPW and HPWcatal

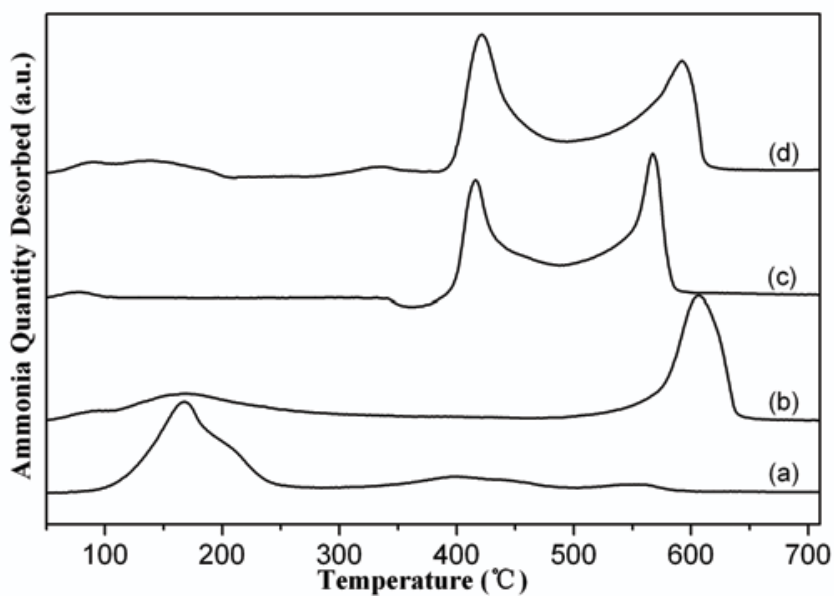

Figure 4. No-NH3-TPD profiles of HPW(a) and HPW-catal(c), NH3-TPD profiles of HPW(b) and HPW-catal(d)

In order to analyse the catalyst further, the $\mathrm{NH}_{3}$-TPD was empolyed to detect the acid sites on the catalyst. Before the experiments, the TPD was carried out without $\mathrm{NH}_{3}\left(\mathrm{No}^{-} \mathrm{NH}_{3}-\mathrm{TPD}\right)$. It is found that the HPW shows an obvious peak before $200^{\circ} \mathrm{C}$ attributed to the presence of water. The HPW-catal shows an weak peaks before $200^{\circ} \mathrm{C}$ due to its hydrophobic nature and a strong peak between $550-640^{\circ} \mathrm{C}$ contribute to the destructure of lurylamine. For 
the $\mathrm{NH}_{3}$-TPD analysis, HPW exibits two peaks around $400{ }^{\circ} \mathrm{C}$ and $600{ }^{\circ} \mathrm{C}$ which was attributed to mediate strong acid site and a strong acid site respectively. Judging from the area of the peaks, the amount of mediate acid sites is relative equal to the strong one. Intrestingly, after the modification, the HPW-catal also show two obvious peaks atrributed to mediate strong acid site and strong acid site. Combined with the analysis of $\mathrm{No}_{-} \mathrm{NH}_{3}-\mathrm{TPD}$, the HPWcatal shows more mediate strong acid sites after modification due to the reaction between the strong acid site and HPW. The EA analysis was empolyed to evaluate the amount of the organic molecule in the structure of HPW-catal. It is turned out that the nitrogen content was about $0.593 \mathrm{mmol} / \mathrm{g}$. By theoretical caculation, the nitrogen content of HPW-catal should be $0.329,0.614$, $0.871 \mathrm{mmol} / \mathrm{g}$ corresponding to $1: 1,2: 1,3: 1$ reactant ratio for lurylamine/HPW. That is to say, to react with the proton of $1 \mathrm{mmol} \mathrm{HPW}$, at least, it needs $3 \mathrm{mmol}$ lurylamine. So, in our experiments, the accounts of HPW were excess, which should explain the strong acid site of HPW-catal since only part of $\mathrm{H}^{+}$ions in HPW had combined with lurylamine.

To evaluate the catalytic performance of the lurylamine modified heteropoly acid in the water, the hydrolysis reaction of sucrose and ethyl acetate was carried out. For the hydrolysis of sucrose(Figure 5), two kinds of products (glucose and fructose) were generated.the yield of glucose was used to measure the catalytic activity. The results of glucose yield for the hydrolysis of sucrose catalyzed by HPW-catal, AWP, AM-15 and HZSM-5 were summarized in Table 1. It is found that the glucose yield of $76 \%$ can be obtained after $0.5 \mathrm{~h}$ and $100 \%$ is got after $2 \mathrm{~h}$, when the HPW-catal served as the catalyst and the reaction temperature was $75^{\circ} \mathrm{C}$ (Table 1 , entry 1-5). The temperture effect on the reactions was also evaluated in the experiments (Table 1, entry 6-10). The results indicated that the optimal conditions of sucrose hydrolysis over HPW-catal was $2 \mathrm{~h}$, $75{ }^{\circ} \mathrm{C}$ with $100 \%$ glucose yield under microwave. It should be pointed out that no glucose yield was obtained for the reaction without any catalysts (Table 1, entry14) under such that conditions, which indicated that the HPWcatal indeed served as catalyst during the reaction. For the comparation, the catalyst of AWP, AM-15 and HZSM-5 were also employed to catalyse the reaction under the same condition. The results showed that the glucose yield was $4 \%, 100 \%$ and $56 \%$ respectively (Table 1 , entry $12-$ 14). That is to say the activity of HPW-catal is the same as commercial AM-15, and higher than AWP and HZSM-5.

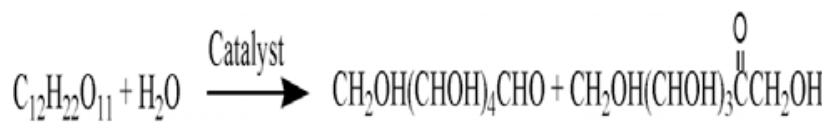

\section{$\begin{array}{lll}\text { Sucrose } & \text { Glucose } & \text { Fructose }\end{array}$}

Figure 5. Process for the hydrolysis of sucrose
Table 1. Results of sucrose hydrolysis over catalysts under microwave condition

\begin{tabular}{|c|c|c|c|c|}
\hline Entry & Catalyst & $\begin{array}{l}\text { Time } \\
/(\mathrm{h})\end{array}$ & $\begin{array}{l}\text { Temperature } \\
/\left({ }^{\circ} \mathrm{C}\right)\end{array}$ & $\begin{array}{l}\text { Glucose } \\
\text { yield } /(\%)\end{array}$ \\
\hline 1 & & 0.5 & & 76 \\
\hline 2 & & 1 & & 88 \\
\hline 3 & & 1.5 & & 94 \\
\hline 4 & & 2 & 75 & 100 \\
\hline 5 & & 2.5 & & 100 \\
\hline 6 & & 2 & 60 & 22 \\
\hline 7 & & 2 & 65 & 56 \\
\hline 8 & & 2 & 70 & 88 \\
\hline 9 & & 2 & 75 & 100 \\
\hline 10 & & 2 & 80 & 100 \\
\hline 11 & AWP & 2 & 75 & 4 \\
\hline 12 & AM-15 & 2 & 75 & 100 \\
\hline 13 & HZSM-5 & 2 & 75 & 56 \\
\hline 14 & - & 2 & 75 & 0 \\
\hline
\end{tabular}

The hydrolysis of ethyl acetate in the aqueous solution was also employed to evaluated the performance of catalyst (Table 2). Alcohol yield of 95\% can be obtained within $1.5 \mathrm{~h}$ at $70^{\circ} \mathrm{C}$ under the microwave for the HPWcatal. As comprison, AWP, AM-15, HZSM-5 and dilute sulphuric acid were also used to catalyzed the reaction(Table 2, entry 6-11). Under the same condition the alcohol yield for the calalyst of AM-15, HZSM-5 and AWP is $89.7 \%, 80.4 \%$ and $2.8 \%$. Interestingly, the HPWcatal showed higher activity on hydrolysis of ethyl acetate than HZSM-5, AWP and even AM-15. The most important reason is believed that hydrolysis of ethyl acetate catalyzed by HPW-catal in water is a homogenous reaction while others were heterogenous reactions. It should be note that the hydrophobic HPW-catal can be dissolved in ethyl acetate before reacting. In the water reaction system, the ethyl acetate is a separation phase due to the hydrophobic nature. During the reaction, the HPWcatal mixs very well with ethyl acetate, when the hydrolysis takes place, the products alchohol and acetic acid will get into water directly, the equilibrium of reaction will change. When the reaction reached equilibrioum, the ethyl acetate was almost used out, the HPW-catal was automatically separated due to its hydrophobic nature. It is well known that the inorganic miner acid such as sulphuric acid can also give a high conversion of hydrolysis of ethyl acetate. But the seperation and recycle problems for $\mathrm{H}_{2} \mathrm{SO}_{4}$ was still not solved, while HPW-catal precipitated from the system spontaneously after reaction and could be used repeatedly.

Figure. 6 shows the plots of alcohol yield versus time for the hydrolysis of ethyl acetate at $70{ }^{\circ} \mathrm{C}$ catalyzed by the HPW-catal under microwave irradiation and conventional heating (oil bath), rspectively. Evidently, compared with the conventional heating, the microwave 
irradiation significantly accelerated the hydrolysis reaction and increased the yield of alcohol. Under conventional heating, the maximum yield of alcohol was $63 \%$ after $5 \mathrm{~h}$ for reaction, while the alcohol yield of about $75 \%$ can be obtained under microwave irradiation for only $0.5 \mathrm{~h}$. Additionally, the maxium yield of $95 \%$ can be reached after irradiation of $1.5 \mathrm{~h}$. The rate of ethyl acetate hydrolysis under the microwave irradation is estimated to be much more 5 times than that under converntional heating. It was believed that the microwave irradiation might activate the participating molecules in the reaction, and improve the effective contraction among molecules, which lead to the acceleration the hydrolysis process.

Table 2. Results of ethyl acetate hydrolysis over catalysts under mcrowave conditions

\begin{tabular}{lllll}
\hline Entry & Catalyst & $\begin{array}{l}\text { Time } \\
/(\mathrm{h})\end{array}$ & $\begin{array}{l}\text { Temperture } \\
/\left({ }^{\circ} \mathrm{C}\right)\end{array}$ & $\begin{array}{l}\text { Alcohol } \\
\text { yield } /(\%)\end{array}$ \\
\hline 1 & & 0.5 & & 75.1 \\
2 & & 1 & 83.5 \\
3 & HPW-catal & 1.5 & 95 \\
4 & $\mathrm{AM}-15$ & 1.5 & 70 & 89.7 \\
5 & $\mathrm{HZSM}-5$ & 1.5 & & 80.4 \\
6 & $\mathrm{AWP}$ & 1.5 & 2.8 \\
7 & $1 \mathrm{mlH}_{2} \mathrm{SO}_{4}$ & 0.5 & & 100 \\
10 & $3 \mathrm{mlH}_{2} \mathrm{SO}_{4}$ & 0.5 & 88.5 \\
11 & $5 \mathrm{mlH}_{2} \mathrm{SO}_{4}$ & 0.5 & 86.6 \\
\hline
\end{tabular}

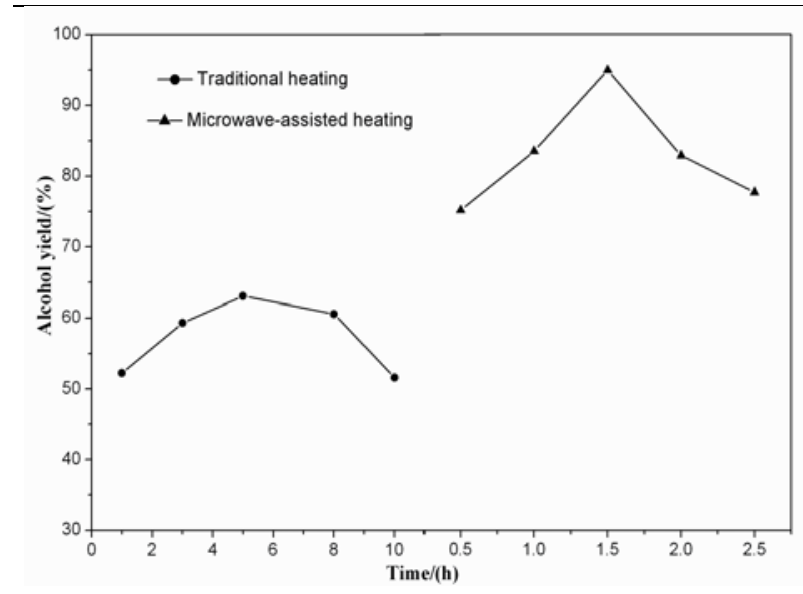

Figure 6. The contrast of alcohol yield between the condition of traditional heating and microwave-assisted heating at $70{ }^{\circ} \mathrm{C}$.

In the aqueous reaction system, the HPW-catal can be recycled easyliy due to the hydrophobic nature. The catalyst was repeated 5 cycles for the hydrolysis of sucrose and ethyl acetate (Figure. 7). It can be seen that after 5 recycles the HPW-catal can still performed high activity on both of the reactions.

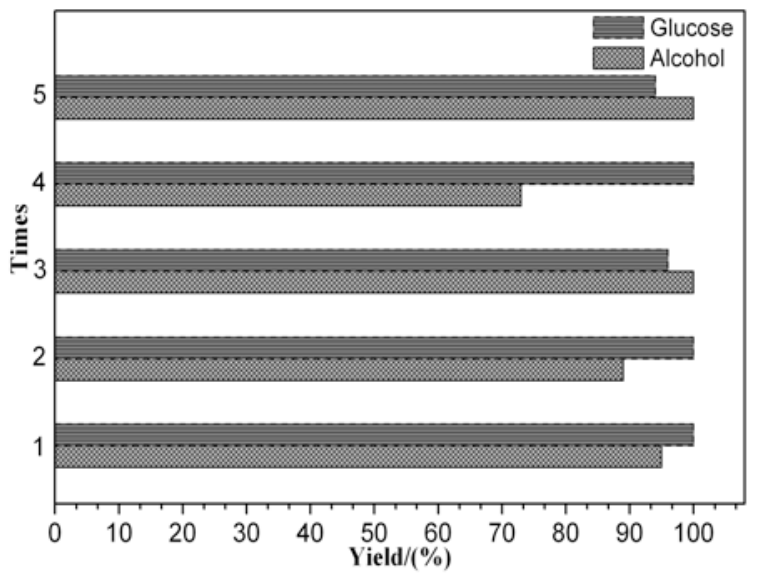

Figure 7. Results of HPW-catal repeatability tested on the two reactions

\section{Conclusions}

In summary, we have demonstrated that a new HPW modified catalyst not only was hydrophobic but maintain high catalytic activity. In the hydrolysis reaction of ethyl acetate, alcohol yield of $95 \%$ was obtained. It's worth mentioning that HPW-catal was soluble in ethyl acetate which was different from other solid acid, so the solution was emulsion with magnetic stirring during the reaction, but precipitated from the system spontaneously after reaction and could be used repeatedly. Glucose yield of $100 \%$ was got by using HPW-catal in the hydrolysis of sucrose, which is same as using AM-15. Additionally, the HPW-catal catalyst showed a stable catalytic activity and can be reused after reaction. Due to the satisfactory catalytic performance, easy availability and its special in dissolubility, HPW-catal shows encouraging prospects for practical uses in hydrolysis reactions.

\section{References}

1. A. Takagaki, C. Tagusagawa, S. Hayashi, M. Hara and K. Domen, "Nanosheets as highly active solid acid catalysts for green chemical syntheses", Energy Environmental Science, 2010, vol. 3, pp. 82-93.

2. A. Corma "Inorganic solid acids and their use in acidcatalyzed hydrocarbon reactions", Chemical Reviews, 1995, vol. 95, pp.559-614.

3. T. Okuhara, "Water-Tolerant solid acid catalysts", Chemical Reviews, 2002, vol. 102, pp.3641-3666.

4. I. V. Kozhevnikov, "Catalysis by heteropoly acids and multicomponent polyoxometalates in liquidphase reactions", Chemical Reviews, 1998, vol. 98, pp.171-198.

5. T. Okuhara, "New catalytic functions of heteropoly compounds as solid acids", Catalysis Today, 2002, vol. 73, pp. 167-176.

6. I. Kozhevnikov, "Heterogeneous acid catalysis by heteropoly acids: Approaches to catalyst deactivation", Journal of Molecular Catalysis A: Chemical, 2009, vol. 305, pp. 104-111. 
7. M. Misono,N. Nojiri, "Recent progress in catalytic technology in japan", Applied catalysis, 1990, vol. 64, pp. 1-30.

8. E. F. Kozhevnikova, E. G. Derouane and I. V. Kozhevnikov, "Heteropoly acid as a novel efficient catalyst for Fries rearrangement", Chemical Communications, 2002, pp. 1178-1179.

9. J. Kaur, K. Griffin, B. Harrison and I. Kozhevnikov, "Friedel-Crafts acylation catalysed by heteropoly acids", Journal of Catalysis, 2002, vol. 208, pp. 448455.

10. H. Firouzabadi, N. Iranpoor and K. Amani, "SolventFree and selective oxidation of hydroxy groups to their corresponding carbonyl functions with ferric nitrate activated by heteropoly acids", Synthesis, 2003, pp. 408-412.

11. J. Tian, J. H. Wang, S. Zhao, C. Y. Jiang, X. Zhang and X. H. Wang, "Hydrolysis of cellulose by the heteropoly acid $\mathrm{H}_{3} \mathrm{PW}_{12} \mathrm{O}_{40}$ " Cellulose, 2010, vol. 17, pp. 587-594.

12. K.-i. Shimizu, H. Furukawa, N. Kobayashi, Y. Itaya and A. Satsuma, "Effects of Brønsted and Lewis acidities on activity and selectivity of heteropolyacidbased catalysts for hydrolysis of cellobiose and cellulose", Green Chemistry, 2009, vol. 11, pp.16271632.

13. R. Palkovits, K. Tajvidi, A. M. Ruppert and J. Procelewska, "Heteropoly acids as efficient acid catalysts in the one-step conversion of cellulose to sugar alcohols", Chemical Communication (Camb), 2011, vol. 47, pp. 576-578.

14. Q. Y. Liu, W. L. Wu, J. Wang, X. Q. Ren and Y. R. Wang, "Characterization of 12-tungstophosphoric acid impregnated on mesoporous silica SBA-15 and its catalytic performance in isopropylation of naphthalene with isopropanol", Microporous and Mesoporous Materials, 2004, vol. 76, pp. 51-60.
15. S. Mukai, L. Lin, T. Masuda and K. Hashimoto, "Key factors for the encapsulation of Keggin-type heteropoly acids in the supercages of Y-type zeolite" Chemical Engineering Science, 2001, vol. 56, pp. 799-804.

16. F. Marme, G. Coudurier and J. C. Védrine, "Acidtype catalytic properties of heteropolyacid H3PW12O40 supported on various porous silicabased materials", Microporous and Mesoporous Materials, 1998, vol. 22, pp. 151-163.

17. M. E. Chimienti, L. R. Pizzio, C. V. Cáceres and M. N. Blanco, "Tungstophosphoric and tungstosilicic acids on carbon as acidic catalysts", Applied Catalysis A: General, 2001, vol. 208, pp. 7-19.

18. W. G. Klemperer,C. G. Wall, "Polyoxoanion chemistry moves toward the future: from solids and solutions to surfaces", Chemical Reviews, 1998, vol. 98, pp. 297-306.

19. J. Tian, C. Fang, M. Cheng and X. Wang, "Hydrolysis of cellulose over CsxH3-xPW12O40 (X $=1-3)$ heteropoly acid catalysts", Chemical Engineering \& Technology, 2011, vol. 34, pp. 482486.

20. L. Zhang, Q. Jin, L. Shan, Y. Liu, X. Wang and J. Huang, "H3PW12O40 immobilized on silylated palygorskite and catalytic activity in esterification reactions", Applied Clay Science, 2010, vol. 47, pp. 229-234.

21. Y. Leng, J. Wang, D. Zhu, Y. Wu and P. Zhao, "Sulfonated organic heteropolyacid salts: Recyclable green solid catalysts for esterifications", Journal of Molecular Catalysis A: Chemical, 2009, vol. 313, pp. 1-6.

22. M. T. Pope, Y. Jeannin and M. Fournier, Heteropoly and isopoly oxometalates, Springer-Verlag Berlin, 1983. 